\title{
Combination of a Novel HDAC 6 Inhibitor ACY-241 and Anti-PD- L1 Antibody Enhances Anti-Tumor Immunity and Cytotoxicity in Multiple Myeloma
}

\author{
Arghya Ray, PhD, Deepika Sharma Das, PhD, Yan Song, PhD, Teru Hideshima, MD, PhD, \\ Yu-Tzu Tai, PhD, Dharminder Chauhan, PhD ${ }^{*}, \#$, and Kenneth C Anderson, MD ${ }^{*}, \#$ \\ The LeBow Institute for Myeloma Therapeutics and Jerome Lipper Myeloma Center, Department \\ of Medical Oncology, Dana-Farber Cancer Institute, Harvard Medical School, Boston, MA
}

\section{Keywords}

Histone deacetylase Inhibitor; Multiple Myeloma; Immunotherapy; Plasmacytoid Dendritic Cells; PD-L-1; ACY-241

\section{Letter to the Editor}

Histone deacetylases (HDACs) are attractive therapeutic targets, and selective HDAC inhibitors (HDACi), alone or in combination with other anti-cancer agents, are promising treatment strategies in multiple myeloma (MM). ${ }^{1-4}$ To date, however, the effect of HDACi on immune regulation in the MM bone marrow (BM) microenvironment, as well as the therapeutic potential of combining selective HDACi with immune-based therapies, is undefined. Recent studies showed that besides histone modification, HDACs also modulate non-histone targets including immune regulatory molecules. ${ }^{5,6}$ For example, blockade of HDACs upregulates immunecheckpoints such as PD-1 ligand (PD-L1). ${ }^{6}$ Moreover, the combination of HDACi with PD-1/PD-L1 checkpoint blockade significantly improves immunotherapy in a murine B16F10 model. ${ }^{6}$ In MM, PD-L1 expression directly correlates with progression of disease, with highest levels in patients with relapsed/refractory MM. ${ }^{7,8}$ Here we utilized an orally bioavalable HDAC6 selective inhibitor ACY-241, currently in clinical trials ${ }^{9}$, to examine whether the combination of ACY-241 with anti-PD-L1 antibody enhances anti-MM immunity in the MM BM microenvironment.

\footnotetext{
*Correspondence: Dharminder Chauhan, Ph.D, Kenneth C. Anderson, M.D.,. Dana-Farber Cancer Institute, M561, 450 Brookline Ave, Boston, MA; Dharminder Chauhan, Ph.D., Dana-Farber Cancer Institute, M561, 450 Brookline Ave, Boston, MA 02215; Dharminder_Chauhan@dfci.harvard.edu; DC: Ph: 617-632-4563; KCA: Ph: 617-632-2144; Fax\#: 617.632.2140. ${ }^{\#} \mathrm{DC}$ and $\mathrm{KC} A$ are Joint Senior authors

Conflicts of Interest disclosure. K.C.A. is an advisor for Celgene, Millennium Pharmaceuticals, and Gilead Sciences and is a Scientific Founder of OncoPep, and C4 Therapeutics. Other authors have no competing financial interests.

'Supplementary information is available at Leukemia's website'

Publisher's Disclaimer: This is a PDF file of an unedited manuscript that has been accepted for publication. As a service to our customers we are providing this early version of the manuscript. The manuscript will undergo copyediting, typesetting, and review of the resulting proof before it is published in its final citable form. Please note that during the production process errors may be discovered which could affect the content, and all legal disclaimers that apply to the journal pertain.
} 
For our studies, we utilized a co-culture model of immune effector cells (plasmacytoid dendritic cells (pDCs), T cells, NK cells) and MM cells. ${ }^{7,10}$ Specifically, we have identified that interactions of MM cells with innate and adaptive immune cells in the BM milieu confer immune suppression via PD-1/PD-L1 signaling axis (Fig 1A:Schema). ${ }^{7,10}$ This is exemplified in our studies demonstrating a novel functional interaction between pDCs ( $\mathrm{Lin}^{-}$ MHC-II ${ }^{+} \mathrm{CD} 123^{+} \mathrm{CD}^{+}$CD303/BDCA-2 $\left.{ }^{+} \mathrm{CD} 304 / \mathrm{BDCA}^{+}\right)$and MM cells, implicating pDCs in both $\mathrm{MM}$ pathogenesis and immune suppression. We showed that increased numbers of pDCs are present in MM patient BM versus normal BM. Importantly, $\mathrm{pDCs}$ in MM BM are relatively resistant to current therapies; protect tumor cells from therapyinduced cytotoxicity; promote tumor growth and survival; and confer immune dysfunction. 7,10 Using our co-culture model, we further delineated the immunosuppressive mechanism(s) that play a role during pDC-T-NK cell interactions with tumor cells. Specifically, we found that: 1) MM cells and pDCs expressed high surface levels of PD-L1, whereas T cells showed high PD-1 levels; and 2) PD-L1 on MM pDCs suppresses PD-1 expressing $\mathrm{T}$ cell and NK cell immune function; and 3) blockade of PD-L1-PD-1 signaling axis by anti-PD-L1 Ab generates MM-specific CD8+ CTL activity, as well as enhances NKcell-mediated MM cell cytolytic activity. Importantly, our co-culture model allows for evaluation of the effect of anti-MM drugs on immune regulatory molecules expressed on immune cells and tumor cells, as well as provides a platform for validating all immunebased combination strategies, especially in the context of immune cells within the MM BM microenvironment. In the current study, we utilized these models to specifically examine whether the combination of ACY-241 with anti-PD-L1 Ab increases anti-tumor immunity and cytotoxicity in MM.

We first evaluated whether the combination of ACY-241 and anti-PD-L1 Ab enhances MMpatient NK cell-mediated cytolytic activity. For these studies, we utilized low concentration of ACY-241 that do not significantly affect the viability of pDCs, T cells, or NK cells (Supplementary Figure 1 A-C). Freshly isolated NK cells from MM patient BM $(n=7)$ were co-cultured with autologous pDCs in the presence of anti-PD-L1 Ab $(5 \mu \mathrm{g} / \mathrm{ml})$, ACY-241 $(0.2 \mu \mathrm{M})$, or ACY-241 plus anti-PD-L1 Ab for 5 days. Cells were washed and resuspended in complete medium without drugs and cultured with autologous MM cells prestained with Celltrace violet for $24 \mathrm{~h}$, followed by 7 -AAD staining and quantification of MM cell lysis by FACS. The combination of ACY-241 and anti-PD-L1 Ab triggered a more robust NK-cell mediated cytotlytic acivity against MM cells than either agent alone (Figure $1 \mathrm{~B}$ and Figure 1C). No significant NK cell-mediated cytotoxicity was noted in the absence of pDCs in these experiments (Supplementary Figure 2B), confirming a key role of pDCs in NK cell functioning in response to treatment with ACY-241 and/or anti-PD-L1 Ab. To further confirm our findings, we next performed analysis of CD107a as a marker of NK cell function. MM patient pDCs and autologous NK cells were treated with ACY-241, anti-PDL1 Ab, or ACY-241 plus anti-PD-L1 Ab, followed by degranulation assay to assess surface $\mathrm{CD} 107$ a expressing $\mathrm{CD}^{-} / \mathrm{CD}^{2} 6^{+} \mathrm{NK}$ cells by multi-parameter flow cytometry. In concert with our finding shown in Figure 1B/1C, ACY-241 plus anti-PD-L1 Ab increases CD107a ${ }^{+}$ degranulated cytolytic NK cells (Supplementary Figure 3).

As in our prior study ${ }^{7}$, anti-PD-L1 Ab alone restored NK-cell-mediated anti-MM activity. Interestingly, treatment of pDC-NK cells co-cultured with ACY-241 alone triggerred NK 
cell cytolytic activity against MM cells (Figure 1B and Figure 1C). In this context, a recent study showed a role of HDAC6 in the regulation of PD-L1 in melanoma. ${ }^{11}$ We hypothesized that ACY-241 may downregulate PD-L1/PD-1-mediated NK cell immune suppression during pDC-NK cell interactions. Indeed, ACY-241 significantly decreases PD-L1 expression on pDCs (Supplementary Figure 5), which in turn attentuates PD-L1/PD-1 mediated NK suppression and thereby enhances NK cell-mediated MM cell cytotoxicity. Although not examined here, other possibilities may account for ACY-241 activity. Similar to immunomodulatory agent Lenalidomide, ACY-241 may skew effector cytokine (IFN- $\gamma$; $\mathrm{TNFa}$ ) production towards enhancing NK cell anti-MM activity. Alternatively ACY 241 may act as reported for HDACi LBH589, which upregulates activation markers CD69 on NK cells. ${ }^{12}$ Finally, ACY-241 may enhance NK-cell anti-MM activity by altering receptorligand (e.g. NKG2D, or KIR) expression- mediated tumor cell killing. These issues remains to be examined. Nonetheless, we here show that: 1) pDC-MM cell interactions modulate NK cell cytotoxic activity; 2) combined ACY-241 and anti-PD-L1 Ab enhances MM-patient pDC-induced NK cell-mediated cytolytic activities against autologous MM cells; 3 ) ACY-241 or anti-PD-L1 alone have the ability to stimulate NK cell effector function, albiet to a lesser extent than in combination; and 4) ACY-241 is an immune-modulating agent since it triggers significant NK cell anti-MM activity.

A recent study showed that a HDAC6i ricolinostat triggers T cell activation and APC function. ${ }^{13}$ We therefore next examined whether ACY-241, anti-PD-L1, or their combination triggers the generation of MM-specific cytotoxic T lymphocyte (CTLs) ex vivo. MM patient $\mathrm{BM} \mathrm{C} 8^{+} \mathrm{T}$ cells $(\mathrm{n}=7)$ were co-cultured with autologous pDCs (pDC:T; 1:10 ratio) in the presence of anti-PD-L1 Ab, ACY-241, or ACY-241 plus anti-PD-L1 Ab for 5 days. Cells were washed and resuspended in complete medium without drugs and cultured with autologous MM cells that were pre-stained with CellTracker Green for $24 \mathrm{~h}$, followed by 7 AAD staining and quantification of CTLs-mediated MM cell lysis by FACS. The combination of ACY-241 and anti-PD-L1 Ab triggered a more robust autologous MMspecific $\mathrm{CD} 8^{+} \mathrm{CTL}$ activity than either agent alone, evidenced by decreased number of viable CellTracker Green-positive MM cells (Figure 2A and Supplementary Figure 4). MM cells were also cultured alone without immune effector cells for the same time period, and data obtained from flow analysis were used for normalization to account for the spontaneous MM cells death. We next performed CD107a degranulation assays to confirm the generation of CD8+ T-cell cytolytic activity. MM patient pDCs were co-cultured with autologous $\mathrm{T}$ cells and treated with ACY-241, anti-PD-L1 Ab, or ACY-241 plus anti-PD-L1 Ab, followed by degranulation assay to measure $\mathrm{CD} 107 \mathrm{a}$ on the cell surface of activated $\mathrm{CD} 3^{+} / \mathrm{CD} 8^{+} \mathrm{T}$ cells using FACS. In agreement with our findings from CTL assays (Figure 2A and Supplementary Figure 4), a more robust increase in surface CD107 $\mathrm{a}^{+}$cytotoxic $\mathrm{T}$ cells was noted upon treatment with combined ACY-241 and anti-PD-L1 Ab versus either ACY-241 or anti-PD-L1 Ab alone (Figure 2B). No significant MM patient CTL-activity was noticed in the absence of pDCs (Supplementary Figure 2C).

Collectively, our data show that combination of selective HDAC6 inhibitor ACY-241 with anti-PD-L1 Ab triggers both CTLs- and NK cells-mediated MM cell killing (Supplementary Figure 6, Supplementary Figure 7; Schema). Mechanistic studies show that combined activity of ACY-241 and anti-PD-L1 is due to downregulation of immunosuppressive PD- 
L1/PD-1 signaling during pDC-T cells and pDC-NK cells interactions. It is likely that besides blockade of PD-L1/PD-1, this combination regimen also abrogates other immunosuppressive mechanisms and/or activates immune-activating pathways among pDCs, $\mathrm{NK}$, or T cells that together contribute in restoration of anti-MM immunity. Ongoing studies are focused on investigating these immune mechanism(s).

Finally, recent data indicate potential toxicity associated with checkpoint inhibitors in MM. However, in contrast to inhibitor of checkpoint PD-1 (pembrolizumab), clinical trials of antiPD-L1 Abs to date show safety, without any significant adverse events in MM patients. ${ }^{14}$ Moreover, a recent study found a better response rate in patients with higher PD-L1 levels on MM cells, and PD-1 expression on lymphocytes correlated weakly with progression-free survival in a small subset of patients. ${ }^{15}$ These findings, coupled with our data, support translation of combined ACY-241 and anti-PD-L1 Ab into clinical trials to restore immune function, enhance MM cytotoxicity, and improve patient outcome in MM.

\title{
Supplementary Material
}

Refer to Web version on PubMed Central for supplementary material.

\section{Acknowledgments}

Grant Support This investigation was supported by National Institutes of Health Specialized Programs of Research Excellence (SPORE) grant P50100707, RO1 CA207237 (DC, and KCA), and RO1 CA050947. K.C.A. is an American Cancer Society Clinical Research Professor.

\begin{abstract}
Authors' contributions AR performed the experiments, designed research, analyzed data, and wrote the manuscript; YS, TH, DSD helped in flow cytometry; DC designed research, analyzed data, and wrote the manuscript; and KCA analyzed data and wrote the manuscript.
\end{abstract}

Conflicts of Interest disclosure. K.C.A. is an advisor for Celgene, Millennium Pharmaceuticals, and Gilead Sciences and is a Scientific Founder of OncoPep, and C4 Therapeutics. Other authors have no competing financial interests.

\section{References}

1. Santo L, Hideshima T, Kung AL, Tseng J-C, Tamang D, Yang M, et al. Preclinical activity, pharmacodynamic, and pharmacokinetic properties of a selective HDAC6 inhibitor,ACY-1215, in combination with bortezomib in multiple myeloma. Blood. 2012; 119(11):2579-2589. [PubMed: 22262760]

2. Amengual J, Johannet P, Lombardo M, Zullo K, Hoehn D, Bhagat G, et al. Dual Targeting of Protein Degradation Pathways with the Selective HDAC6 Inhibitor ACY-1215 and Bortezomib Is Synergistic in Lymphoma. Clin Cancer Res. 2015; 21(20):4663-4675. [PubMed: 26116270]

3. Mishima Y, Santo L, Eda H, Cirstea D, Nemani N, Yee AJ, et al. Ricolinostat (ACY-1215) induced inhibition of aggresome formation accelerates carfilzomib-induced multiple myeloma cell death. British Journal of Haematology. 2015; 169(3):423-434. [PubMed: 25709080]

4. Dasmahapatra G, Patel H, Friedberg J, Quayle SN, Jones SS, Grant S. In Vitro and In Vivo Interactions between the HDAC6 Inhibitor Ricolinostat (ACY1215) and the Irreversible Proteasome Inhibitor Carfilzomib in Non-Hodgkin Lymphoma Cells. Mol Cancer Ther. 2014; 13(12):28862897. [PubMed: 25239935]

5. Woan KV, Sahakian E, Sotomayor EM, Seto E, Villagra A. Modulation of Antigen Presenting Cells by HDAC inhibitors: implications in autoimmunity and cancer. Immunol Cell Biol. 2012; 90(1):5565. [PubMed: 22105512] 
6. Woods DM, Sodre' AL, Villagra A, Sarnaik A, Sotomayor EM, Weber J. HDAC Inhibition Upregulates PD-1 Ligands in Melanoma and Augments Immunotherapy with PD-1 Blockade. Cancer Immunol Res. 2015; 3(12):1375-1385. [PubMed: 26297712]

7. Ray A, Das DS, Song Y, Richardson P, Munshi NC, Chauhan D, et al. Targeting PD1-PDL1 immune checkpoint in plasmacytoid dendritic cell interactions with T cells, natural killer cells and multiple myeloma cells. Leukemia. 2015; 29(6):1441-1444. [PubMed: 25634684]

8. Görgün G, Samur MK, Cowens KB, Paula S, Bianchi G, Anderson JE, et al. Lenalidomide Enhances Immune Checkpoint Blockade-Induced Immune Response in Multiple Myeloma. Clin Cancer Res. 2015; 21(20):4607-4618. [PubMed: 25979485]

9. Yee AJ, Bensinger WI, Supko JG, Voorhees PM, Berdeja JG, Richardson PG, et al. Ricolinostat plus lenalidomide, and dexamethasone in relapsed or refractory multiple myeloma: a multicentre phase 1b trial. Lancet Oncol. 2016; 17(11):1569-1578. [PubMed: 27646843]

10. Chauhan D, Singh AV, Brahmandam M, Carrasco R, Bandi M, Hideshima T, et al. Functional interaction of plasmacytoid dendritic cells with multiple myeloma cells: a therapeutic target. Cancer Cell. 2009; 16(4):309-323. [PubMed: 19800576]

11. Lienlaf M, Perez-Villarroel P, Knox T, Pabon M, Sahakian E, Powers J, et al. Essential role of HDAC6 in the regulation of PD-L1 in melanoma. Mol Oncol. 2016; 10(5):735-750. [PubMed: 26775640]

12. Klein JM, Henke A, Sauer M, Bessler M, Reiners KS, Engert A, et al. The histone deacetylase inhibitor LBH589 (panobinostat) modulates the crosstalk of lymphocytes with Hodgkin lymphoma cell lines. PLoS One. 2013; 8(11):e79502. [PubMed: 24278143]

13. Adeegbe D, Liu Y, Lizotte PH, Kamihara Y, Aref AR, Almonte C, et al. Synergistic Immunostimulatory Effects and Therapeutic Benefit of Combined Histone Deacetylase and Bromodomain Inhibition in Non-small Cell Lung Cancer. Cancer Discov. 2017 Apr 13. pii: CD-16-1020 [Epub ahead of print].

14. Kumar SK. Checking in: T cells against multiple myeloma. Blood. 2017; 130(10):1175-1176. [PubMed: 28882832]

15. Badros A, Hyjek E, Ma N, Lesokhin A, Dogan A, Rapoport AP, et al. Pembrolizumab, pomalidomide and low dose dexamethasone for relapsed/refractory multiple myeloma. Blood. 2017; 1410:1189-1197. 
Patient MM-BM:Autologous pDC/ NK/MM cells

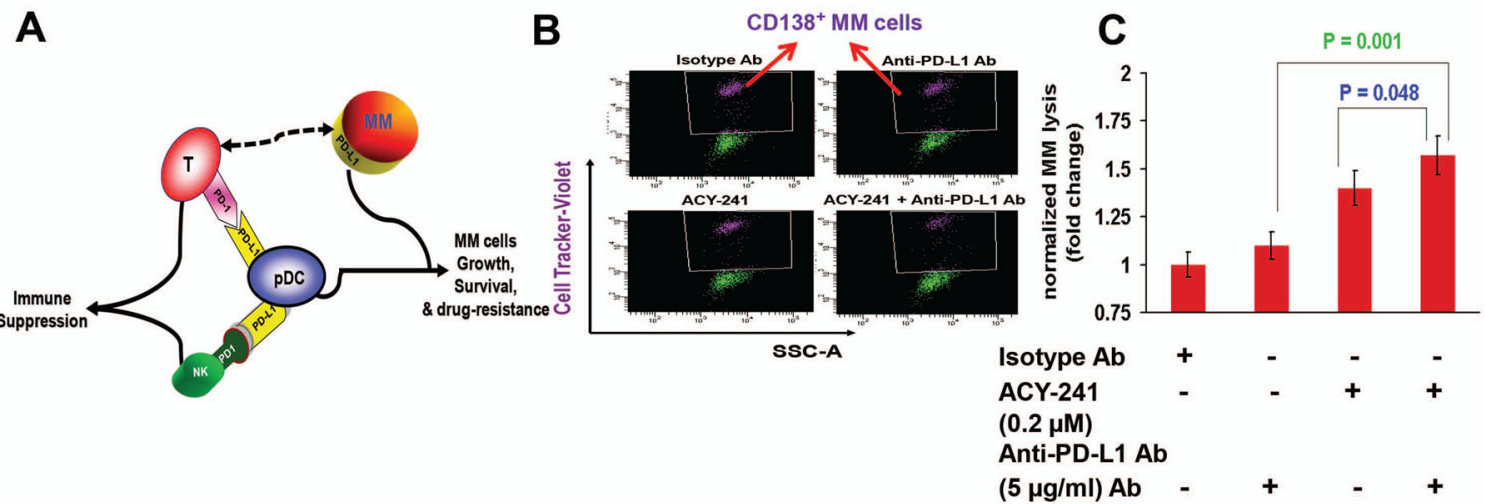

Figure 1. Combination of ACY-241 and anti-PD-L1 enhances MM-patient NK cell-mediated cytolytic activity against patient autologous MM cells

(A) Model depicting the role of plasmacytoid dendritic cells (pDCs) in both conferring $\mathrm{T}$ cell and NK cell immune suppression via PD-L1/PD-1; as well as induction of MM cell growth, survival, and drug-resistance. (B) Freshly isolated NK cells from MM patient BM (n $=7)$ were co-cultured with autologous pDCs (1:10 pDC:NK ratio) in the presence of antiPD-L1 Ab $(5 \mu \mathrm{g} / \mathrm{ml})$, ACY-241 $(0.2 \mu \mathrm{M})$, or ACY-241 plus anti-PD-L1 Ab for 5 days. Cells were then washed and resuspended in complete medium without drugs and cultured with autologous MM cells pre-stained with CellTrace violet (10:1 NK:MM ratio) for 24h, followed by 7-AAD staining and quantification of MM cell lysis by FACS. The decrease in number of viable CellTrace Violet-positive MM cells is shown in a reresentative plot. (C) Bar graph Quantification of NK-mediated MM cell lysis using data obtained in panel B. The fold change was obtained after normalization with control (Data obtained from $7 \mathrm{MM}$ patient $\mathrm{BM}$ samples; mean $\pm \mathrm{SD}$; $\mathrm{p}$ < 0.05). MM cells were also cultured alone without immune effector cells for $24 \mathrm{~h}$, and data obtained from flow analysis were used for normalization to account for the spontaneous MM cells death. In the absence of immune effector cells, the spontaneous MM cell death is $5 \% \pm 1 \%$. This value was subtracted from the percentage of lysed MM cells all treatment groups (Effector/Target coculture; in the presence of isotype Ab, anti-PD-L1 Ab, ACY-241, or anti-PD-L1 Ab plus ACY-241). Finally, the subtracted value is presented as normalized MM lysis (fold change versus isotype Ab control; without anti-PD-L1 Ab and ACY-241). Normalized MM lysis (fold change) is calculated as follows: Normalized MM lysis (fold change $)=[($ NK-induced MM lysis) - spontaneous MM lysis)/((NK-induced MM lysis) $)_{\text {Isotype }}$ - spontaneous MM lysis)]. 


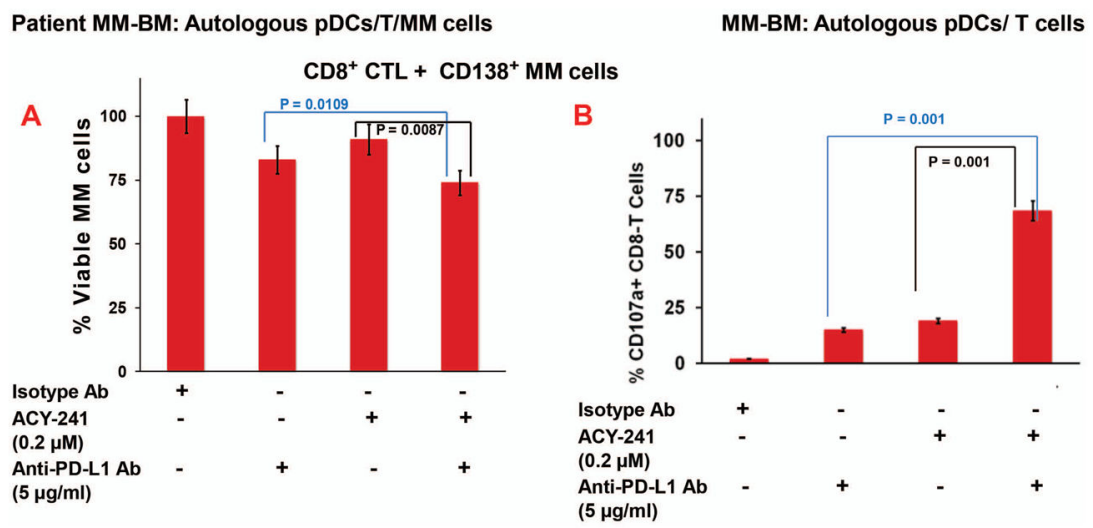

Figure 2. Combination of ACY-241 and anti-PD-L1 enhances MM-specific autologous Cytotoxic T Lymphocyte (CTL) activity MM patient $\mathrm{BM} \mathrm{C} 8^{+} \mathrm{T}$ cells $(\mathrm{n}=7)$ were co-cultured with autologous pDCs (pDC:T; 1:10 ratio) in the presence of anti-PD-L1 Ab, ACY-241, or ACY-241 plus anti-PD-L1 Ab for 5 days Cells were then washed and resuspended in complete medium without drugs and cultured with autologous MM cells (T/MM; 20:1 ratio) that were prestained with CellTracker Green for $24 \mathrm{~h}$, followed by 7 -AAD staining and quantification of CTLsmediated MM cell lysis by FACS. The decrease in number of viable CellTracker Greenpositive MM cells was quantified. (A) Bar graph Quantification of CD8 ${ }^{+}$CTLs-mediated MM cell lysis (Data obtained from 7 MM patient BM samples; mean \pm SD; $p<0.05$ ). MM cells were also cultured alone without immune effector cells for $24 \mathrm{~h}$, and data obtained from flow analysis were used for normalization to account for the spontaneous MM cells death. In the absence of immune effector cells, the spontaneous MM cell death is $5 \% \pm 1 \%$. This value was subtracted from the percentage of lysed MM cells all treatment groups (Effector/ Target coculture; in the presence of isotype Ab, anti-PD-L1 Ab, ACY-241, or anti-PD-L1 Ab plus ACY-241). Finally, the subtracted data is presented as percent of viable cells (\%viable), normalized versus isotype Ab, in the absence of anti-PD-L1 Ab and ACY-241. \% Viable cell is presented as follows: \% Viable cells $=[1-((\mathrm{MM}$ cells after CTL lysis- spontaneous MM lysis $)] /\left[1-\left((\text { MM cells after CTL lysis })_{\text {isotype }}\right.\right.$ ( (spontaneous MM lysis))] X 100 (B) MM patient pDCs and autologous T cells (1:10 pDC/T ratio) were treated with ACY-241 (0.2 $\mu \mathrm{M})$, anti-PD-L1 Ab $(5 \mu \mathrm{g} / \mathrm{ml})$, or ACY-241 (0.2 $\mu \mathrm{M})$ plus anti-PD-L1 Ab $(5 \mu \mathrm{g} / \mathrm{ml})$, followed by degranulation assay to measure surface CD107a ${ }^{+} \mathrm{T}$ cells by multi-parameter flow cytometry. The plot shows percentage of surface CD107a+ $\mathrm{T}$ cells, indicating degranulated cytotoxic T cells (Data obtained from 7 MM patient BM samples; mean \pm SD; $\mathrm{p}<0.05)$. 